\title{
A Teoria da Decisão Bayesiana na Estratégia Mercadológica Paulo Clarindo Goldschmidt*
}

1. Componentes da Teoria de Decisão Bayesiana. 2. Um exemplo Mercadológico: Marte S.A. 3. Passos para a Resolução do Problema. 4. Dificuldade na Estimativa das Probabilidades a Priori. 5. Conclusão.

A Teoria de Decisão Bayesiana está ìntimamente ligada à tomada de decisão em clima de incerteza. Como já foi exposto em um artigo anterior, existem três possíveis climas em que qualquer decisão deve ser tomada ${ }^{1}$ e várias técnicas são empregadas pelo administrador para auxiliá-lo na escolha da melhor alternativa de ação, dependendo do clima em que esta decisão está inserida. A Teoria de Decisão Bayesiana é uma destas técnicas e nosso objetivo, neste trabalho, é demonstrar como ela pode ser utilizada na análise de problemas mercadológicos em uma emprêsa. Evidentemente, seu campo de ação é muito mais amplo e alcança outras áreas que não a mercadológica, porém, através de analogias ao exemplo que vamos seguir, torna-se fácil ao administrador empregá-la nessas outras áreas de interêsse empresarial.

\section{Componentes da teoria de decisão bayesiana 2}

Para uma melhor sistematização do presente trabalho, faz-se mister uma perfeita compreensão dos elementos que compõem a Teoria de Decisão Bayesiana. Estes elementos são:

* Professor contratado do Departamento de Mercadologia da Escola de Administração de Emprêsas da F.G.V.

1 Goldschmidt, Paulo. Aplicação da Teoria das Decisões a um Problema Mercadológico, Revista de Administração de Emprêsas, vol. 9, n. ${ }^{\circ}$, março 1969 , p. 93 e segs.

2 Alderson, W. e Green, P. E. Planning and Problem Solving in Marketing, Richard D. Irwin, Inc., 1964, p. 107 e 108.

R. Adm. Emp., Rio de Janeiro, $10(1): 65 / 77$, jan./mar. 1970 


\subsection{ANÁLISE A PRIORI}

Frequientemente, em problemas de planejamento mercadológico, o administrador pode contar apenas com seu julgamento em relação à sua atividade; isto significa que a natureza do problema (como por exemplo, proposição de modificação nos preços) ou a amplitude do horizonte do planejamento deve excluir a oportunidade de conseguir informações adicionais com relação ao problema, antes que um compromisso seja assumido. Neste caso, o administrador deve decidir tendo como base sòmente sua experiência passada. Este segmento da Teoria Bayesiana é conhecido como análise a priori.

\subsection{ANÁLISE A POSTERIORI}

A experiência empresarial é passível de constante mutação à medida em que o administrador obtém informações com relação a ações administrativas passadas. A revisão dêstes julgamentos a priori pela incorporação de novas informações é tratada de uma maneira muito especial pela Teoria Bayesiana. Esțe segmento da Teoria é conhecido como análise a posteriori.

\subsection{ANÁLISE PREPOSTERIORI}

Algumas vêzes o administrador terá a opção de adiar sua decisão em função do recebimento de maiores informações relacionadas ao problema. No entanto, para obter estas informações, o administrador incorrerá em custos que podem, também, ocorrer pelo adiamento de sua escolha original. Raramente a informação adicional será perfeitamente confiável, devendo, portanto, avaliar a prudência de adiar sua escolha final (que é chamada decisão terminal) e usar êste adiamento na obtenção de informações adicionais, relacionadas aos estados da natureza desconhecidos comparando-a com a ação imediata, sem a informação adicional. Este segmento da Teoria Bayesiana é chamado de análise preposteriori.

\subsection{DECISÕES SEQÜENCIAIS}

As decisões envolvem, freqüentemente, uma sequiência de ações (incluindo a coleta e análise de informações) a serem realizadas no de- 
correr do tempo. Isto significa que muitas ações, tal como aumento de capacidade de produção, devem ser realizadas sequiencialmente, e alguns cursos de ação atuais podem permitir uma maior flexibilidade na obtenção de escolhas com maiores informações no futuro. Um segmento especial da Teoria Bayesiana, que aplica a análise preposteriori aos processos de decisão em etapas múltiplas, é conhecido como decisões sequienciais em clima de incerteza.

\section{Um exemplo mercadológico: Marte S.A.}

Consideremos o seguinte exemplo para efeito de ilustração: tendo a Marte S.A. conseguido autorização para ser agente de vendas, no Estado do Maranhão, de um grande fabricante de máquinas de contabilidade, cuja fábrica estava sediada em São Paulo, sua diretoria se reuniu a fim de estabelecer um critério para o preenchimento do cargo de gerente de vendas. Alguns elementos da diretoria eram favoráveis à aplicação de um teste ao único candidato ao cargo, enquanto outros eram contra, principalmente em virtude de seu custo - NCr $\$ 5.000,00$ - orçado por uma emprêsa especializada no ramo. Durante a reunião, foram estabelecidas algumas diretrizes que pudessem auxiliá-los na tomada de decisão, e que envolvessem o menor risco para a emprêsa. Dessa forma, ficou resolvido que, caso se optasse pela realização do teste, a decisão de admitir ou não o candidato dependeria de seu quociente de inteligência (QI), dado pelo teste. Além disso, ficou resolvido, também, que sòmente seriam consideradas duas alternativas: a) o candidato tem um QI superior ou igual a $100 \mathrm{e}, \mathrm{b}$ ) o candidato tem um QI inferior a 100.

Nesse momento, foi apresentada uma série de dados históricos, fornecidos pelo fabricante, que correlacionavam o volume de vendas conseguido pelos gerentes de vendas de suas filiais, em diversos períodos, com o QI que êles possuíam. Da análise e tabulação dêsses dados extraíram-se as seguintes conclusões:

a) Nos períodos em que o volume de vendas das filiais era alto, seus respectivos gerentes de vendas sempre tinham QI $\geqslant 100$.

b) Nos períodos em que o volume de vendas das filiais era baixo, seus respectivos gerentes de vendas nunca tinham $Q I \geqslant 100$.

c) Nos períodos em que o volume de vendas das filiais era médio, $60 \%$ de seus respectivos gerentes de vendas tinham QI $\geqslant 100$. 
De acôrdo com o parecer do Diretor de Marketing do fabricante, caso se optasse pela admissão do candidato sem a realização do teste, as probabilidades de as vendas serem altas, médias ou baixas eram aproximadamente iguais, conforme experiência obtida nas várias filiais do fabricante espalhadas por todo o Brasil.

A diretoria da Marte S.A. calcula que o custo de admissão de um gerente de vendas (incluindo a admissão de um corpo de vendedores) estaria por volta de NCr $\$ 120.000,00$ para o período de um ano. Um estudo preliminar de mercado, feito pela Marte S.A., mostrou que a previsão de vendas para o período de um ano seria de 160 unidades, se as vendas fôssem altas; de 100 unidades, se médias, e de 40 unidades, se baixas. Evidentemente, nada seria vendido se não se admitisse o candidato a gerente de vendas. O preço de venda unitário do produto está fixado em NCr\$2.000,00.

\section{Passos para a resolução do problema}

A seguir descreveremos os passos a serem seguidos para a resolução do problema proposto, através da Teoria de Decisão Bayesiana.

\subsection{PRIMEIRo PASSO: CONSTRUÇÃo DA ÁRVORE DE DECISÃo}

O primeiro passo - construção da árvore de decisão - embora não essencial à aplicação da Teoria Bayesiana, visa tão sòmente a uma melhor visualização do problema a ser resolvido. A árvore de decisão apresentada na Figura 1 apresenta tôdas as possibilidades lógicas decorrentes do problema apresentado. ${ }^{3}$

\subsection{SEGUNDO PASSO: ALOCAÇÃo Dòs DADOS DISPONÍvEIS}

O segundo passo - alocação dos dados disponíveis - consiste em se colocar na árvore de decisão tôdas as informações possuídas através da descrição do problema. Assim é que aparecem nos pontos adequados:

3 Para um maior esclarecimento da forma de se trabalhar com árvores de decisão, citamos o trabalho de HAMmoND III, JoHN S. Melhores Decisões com a Teoria da Preferência, publicado no n. ${ }^{\circ} 27$ da $R$. $A$. E., p. 151 e segs. 
FIGURA 1

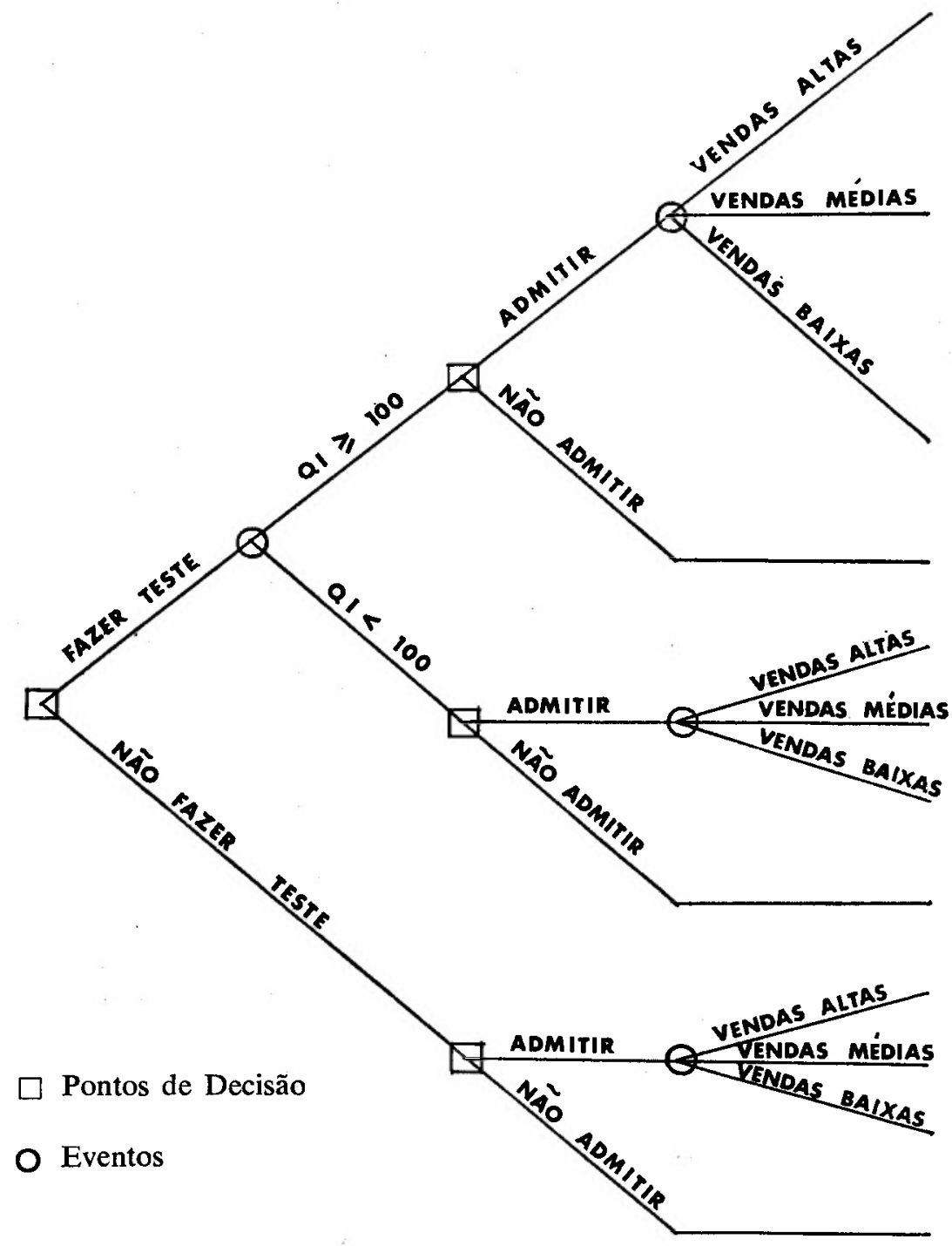

a) Os valôres correspondentes às receitas esperadas, isto é, $\mathrm{NCr} \$$ $80.000,00$ para vendas baixas, $\mathrm{NCr} \$ 200.000,00$ para vendas médias, $\mathrm{NCr} \$ 320.000,00$ para vendas altas;

b) $\mathrm{O}$ custo de admissão do gerente de vendas, ou seja, $\mathrm{NCr} \$$ $120.000,00$ 
c) O custo da aplicação do teste, ou seja, $\mathrm{NCr} \$ 5.000,00$;

d) As probabilidades a priori, ou seja, as probabilidades de as vendas serem altas, médias ou baixas, sem a realização do teste $-1 / 3$, $1 / 3$ e $1 / 3$, respectivamente.

Estes dados podem ser melhor visualizados pela observação da Figura 2.

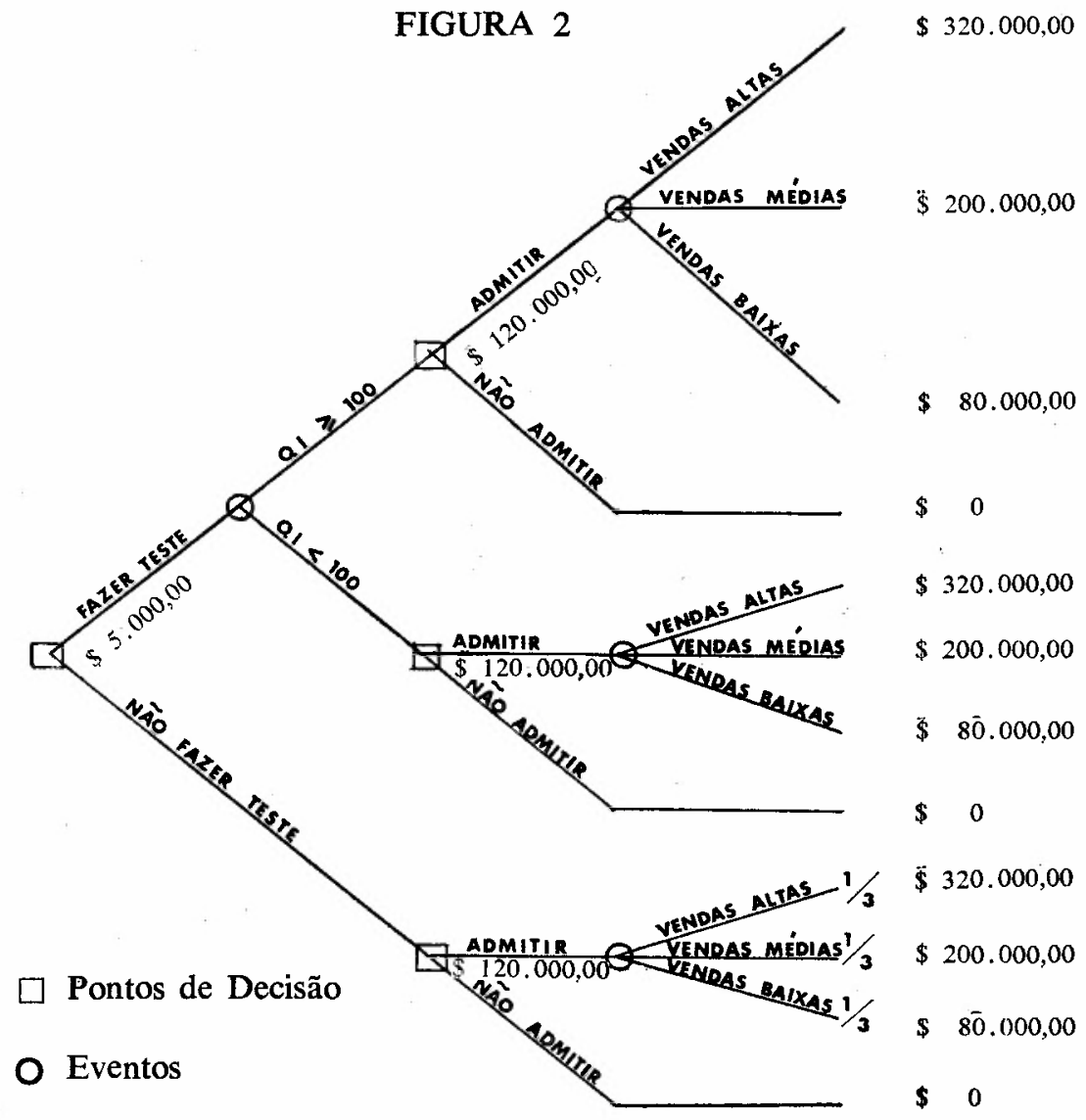

\subsection{TERCEIRO PASSO: OBTENÇÃo DOS DADOS RESTANTES}

O terceiro passo consiste em se obter os dados restantes que não aparecem explìcitamente na descrição do problema, mas que podem ser conseguidos através das informações que nêle constam. Enquanto que as probabilidades de as vendas serem altas, médias ou baixas, sem a 
realização do teste, eram conhecidas, devemos determinar quais seriam elas com sua realização. O aparecimento de informação adicional (como, por exemplo, a de que o candidato tenha um $\mathrm{QI} \geq 100$ ) pode alterar o espaço amostral com o qual estamos trabalhando. Suponhamos, por exemplo, que desejamos saber a probabilidade de as vendas serem altas, dada a informação de que o candidato tenha um QI superior ou igual a 100 . Para tanto, devemos utilizar a seguinte fórmula (Teorema de Bayes) : ${ }^{4}$

$P(V A Y Q I \geq 100)=\frac{P(Q I \geq 100 / V A) . P(V A)}{P(Q I \geq 100 / V A) \cdot P(V A)+P(Q I \geq 100 / V M)}$

onde

$$
. P(V M)+P(Q I \geq 100 / V B) . P(V B)
$$

$\mathrm{P}(\mathrm{VA} / \mathrm{QI} \geq 100)=$ probabilidade condicional de as vendas serem altas, dado que o candidato tenha um $Q I \geq 100$,

$\mathrm{P}(\mathrm{QI}=100 / \mathrm{VA})=$ probabilidade condicional de que o candidato tenha um $Q I \geq 100$, dado que as vendas sejam altas,

$\mathrm{P}(\mathrm{VA}) \quad=$ probabilidade $a$ priori de se ter vendas altas. Todos os outros símbolos que aparecem na fórmula são definidos por analogia. Aplicando-se a fórmula ao exemplo que estamos estudando, teremos:

$$
\begin{aligned}
& \mathrm{P}(\mathrm{VA} / \mathrm{Q} \mathrm{I} \geq 100)=\frac{1 \times 1 / 3}{(1 \times 1 / 3)+(0,60 \times 1 / 3)+(0 \times 1 / 3)} \therefore \\
& \mathrm{P}(\mathrm{VA} / \mathrm{QI} \geq 100)=\frac{1 / 3}{1 / 3+1 / 5}=5 / 8
\end{aligned}
$$

Anàlogamente tercmos:

$$
\begin{aligned}
& \mathrm{P}(\mathrm{VM} / \mathrm{QI} \geq 100)=\frac{0,60 \times 1 / 3}{(1 \times 1 / 3)+(0,60 \times 1 / 3)+(0 \times 1 / 3)}=3 / 8 \\
& \mathrm{P}(\mathrm{VB} / \mathrm{QI} \geq 100)=\frac{0 \times 1 / 3}{(1 \times 1 / 3)+(0,60 \times 1 / 3)+(0 \times 1 / 3)}=0
\end{aligned}
$$

Trabalhando-se da mesma forma com a informação de que o candidato tenha um QI $\geq 100$, podemos calcular quais as probabilidades de as vendas serem altas, médias ou baixas, dada esta nova informação. Assim, aplicando-se o teorema de BAYES, teremos:

$$
\begin{aligned}
& \mathrm{P}(\mathrm{VA} / \mathrm{QI}<100)=0 \\
& \mathrm{P}(\mathrm{VM} / \mathrm{QI}<100)=2 / 7 \\
& \mathrm{P}(\mathrm{VB} / \mathrm{QI}<100)=5 / 7
\end{aligned}
$$

4 A fórmula genérica do teorema de BAYES é a seguinte:

$$
P\left(B_{i} / A\right)=\frac{P\left(A / B_{i}\right) . P\left(B_{i}\right)}{\sum_{j=1}^{n} P\left(A / B_{i}\right) \cdot\left(P\left(B_{j}\right)\right.}
$$


Todos os novos valôres (juntamente com os obtidos anteriormente) são apresentados na Figura 3.

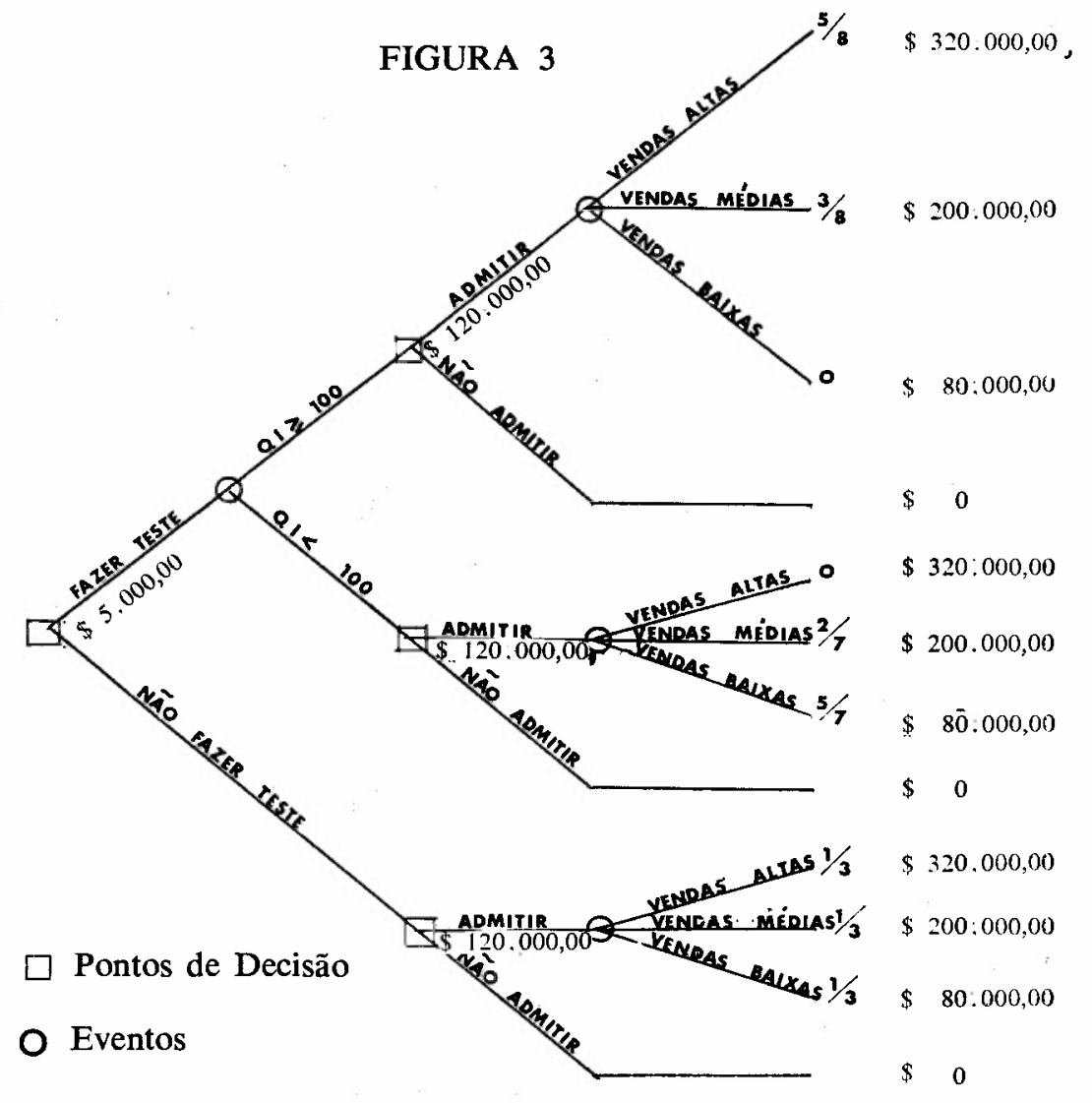

\subsection{QUARTO PASSO: AVALIAÇÃo DAS ALTERNATIVAS}

O quarto passo consiste na avaliação das várias alternativas à disposição do administrador, para que êle escolha a melhor dentre tôdas aquelas que estão à sua disposição. O critério de escolha entre alternativas (ou cursos de ação) sob a abordagem Bayesiana é bastante simples: escolha aquela alternativa que leve ao mais alto valor esperado ou, em outras palavras, a mais alta resultante média ponderada. ${ }^{5}$

5 Green, P. E. e Frank, R. E. A Manager's Guide to Marketing Research, John Wiely \& Sons, Inc., 1969, p. 13. 
Se tomarmos, por exemplo, os entroncamentos finais do tôpo da Figura 3, teremos que o valor esperado do evento que dá origem aos entroncamentos é de NCr $\$ 275.000,00$ (ou seja, NCr $\$ 320.000,00 \times$ $5 / 8+\mathrm{NCr} \$ 200.000,00 \times 3 / 8+\mathrm{NCr} \$ 80.000,00 \times 0$ ). No entanto, a êste valor deve ser subtraído NCr $\$ 120.000,00$ (custo de admissão), restando, pois, $\mathrm{NCr} \$ 155.000,00$. Como êste último valor é claramente maior que o valor esperado sob a alternativa NÃO ADMITIR (NCr\$ 0), o administrador deve escolher a alternativa ADMITIR, eliminando o ramo NÃO ADMITIR na árvore de decisão. $O$ valor dêste ponto de decisão é, portanto, $\mathrm{NCr} \$ 155.000,00$.

De forma análoga, calculamos os outros eventos e pontos de decisão para o restante da árvore. Falta-nos, entretanto, para a complementação do problema, computar a probabilidade de que o candidato tenha um $\mathrm{QI} \geq 100$ versus a probabilidade de que tenha um $\mathrm{QI}<100$. Estas são conhecidas como probabijidades marginais e são obtidas da seguinte forma:

\begin{tabular}{l|c|c|c|c} 
& $\mathrm{P}(\mathrm{VA})$ & $\mathrm{P}(\mathrm{VM})$ & $\mathrm{P}(\mathrm{VB})$ & $\begin{array}{c}\text { Prob. } \\
\text { Mar- } \\
\text { ginal }\end{array}$ \\
\hline $\mathrm{P}(\mathrm{QI} \geq 100)$ & $1 \times 1 / 3=1 / 3$ & $0,60 \times 1 / 3=1 / 5$ & $0 \times 1 / 3=0$ & $8 / 15$ \\
\hline $\mathrm{P}(\mathrm{QI}<100)$ & $0 \times 1 / 3=0$ & $0,40 \times 1 / 3=2 / 15$ & $1 \times 1 / 3=1$ & $7 / 15$ \\
\hline $\begin{array}{l}\text { Probabil. } \\
\text { a priori }\end{array}$ & $1 / 3$ & $1 / 3$ & $1 / 3$ & 1
\end{tabular}

Os valôres dentro de cada célula acima representam a probabilidade conjunta dos eventos considerados. Assim, na primeira célula superior à esquerda, o valor $1 / 3$ foi encontrado da seguinte forma:

$$
\mathrm{P}(\mathrm{QI} \geq 100 / \mathrm{VA}) . \mathrm{P}(\mathrm{VA})=1 \times 1 / 3=1 / 3 .
$$

Este valor representa a probabilidade conjunta da ocorrência de vendas altas e a de que o candidato tenha um $Q I \geq 100$ dada a ocorrência de vendas altas. Os valôres dentro das células restantes foram obtidos de forma análoga. Somando-se horizontalmente as células do quadro anterior obteremos as probabilidades marginais, ou seja,

$\mathrm{P}(\mathrm{QI} \geq 100)=1 / 3+1 / 5+0=8 / 15$;

$\mathrm{P}(\mathrm{QI}<100)=0+2 / 15+1 / 3=7 / 15$

Se somarmos as colunas, obteremos as probabilidades a priori de que tenhamos vendas altas, médias ou baixas (valôres êsses que já conhecíamos). Transpondo-se as probabilidades marginais para a árvore de 
decisão, podemos fazer o restante dos cálculos necessários. A Figura 4 mostra os resultados dos diversos cálculos efetuados, apresentando, ainda, a melhor alternativa para o problema considerado, isto é, não fazer o teste e admitir o candidato, alternativa esta que dá um valor esperado de NCr $\$ 80.000,00$.

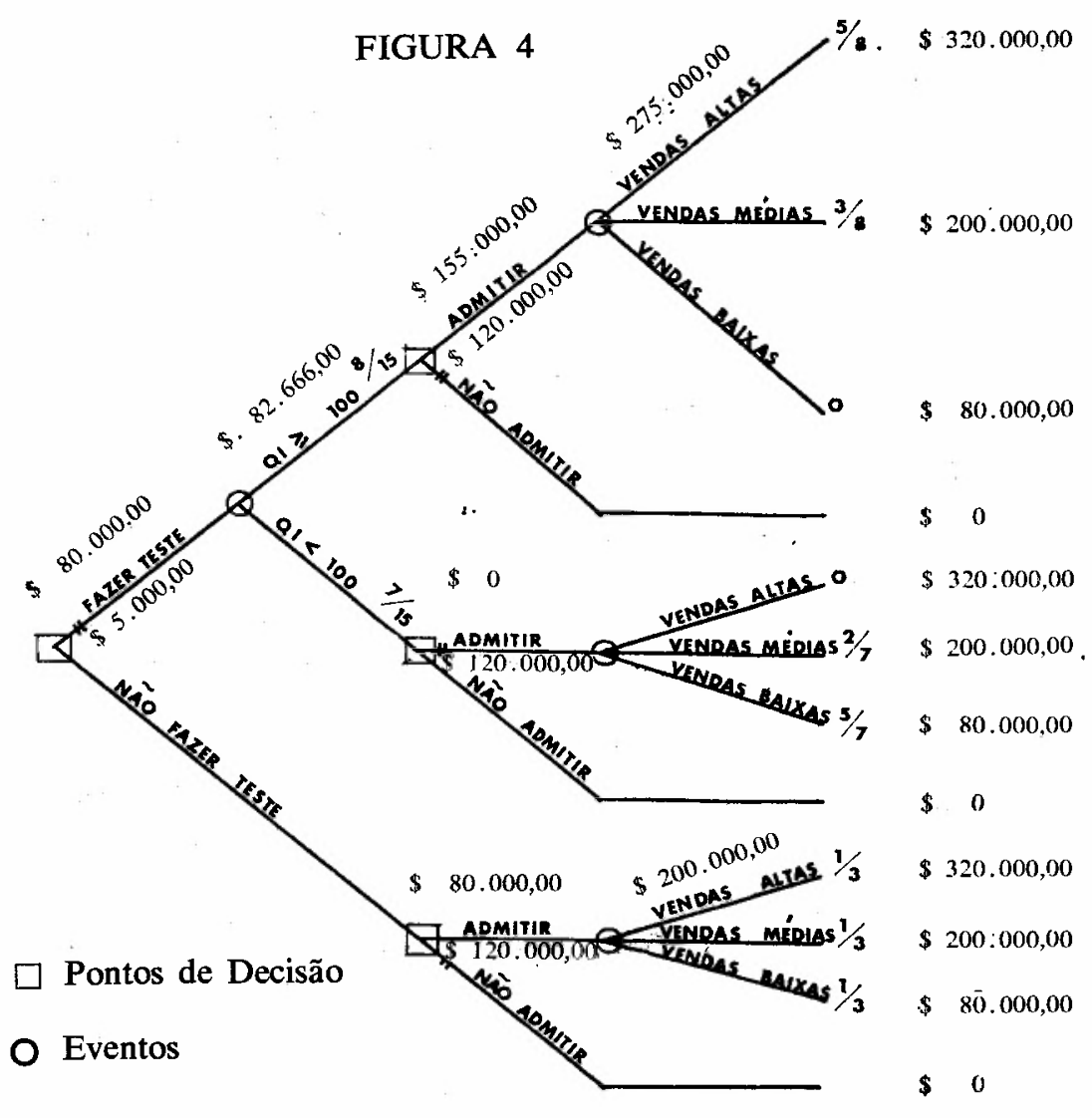

\section{Dificuldade na Estimativa das Probabilidades a Priori}

Uma das maiores dificuldades encontradas pelos administradores está ligada à estimativa das probabilidades $a$ priori. Frequientemente êle supõe um determinado valor, porém, não está perfeitamente convencido de que é um valor certo. Este tópico procura demonstrar como o administrador pode resolver êste problema através da aplicação da análise de sensitividade, que consiste na alteração deliberada das pro- 
babilidades com o intuito de verificar como tais alterações atuam sôbre os diversos valôres esperados. Um exemplo irá esclarecer o que pretendemos demonstrar. Suponha que o administrador tenha três possíveis alternativas de ação e que possam ocorrer sòmente dois estados da natureza, mùtuamente exclusivos, e exaustivos. Da mesma forma, vamos supor que seja possível determinar os pay-offs resultantes da escolha de uma determinada alternativa em conjunto com a ocorrência de um determinado estado da natureza. Estes dados podem ser observados no Quadro 1.

\section{QUADRO 1}

\begin{tabular}{ccc} 
Alternativas & \multicolumn{2}{c}{ Estados da } \\
& $\mathbf{N}_{1}$ & $\mathbf{N}_{2}$ \\
$\mathbf{A}_{1}$ & $\$ 500$ & $\$ 400$ \\
$\mathbf{A}_{2}$ & $\$ 700$ & $\$ 200$ \\
$\mathbf{A}_{3}$ & $\$ 600$ & $\$ 100$
\end{tabular}

No Quadro 1, os pay-offs representam lucro líquido (evidentemente, não é necessário que a medida seja sempre essa; podemos trabalhar com pay-offs que representem custo unitário, lucro unitário, custo total, etc.). Assim, se $o$ administrador escolhe alternativa $A_{1}$, na ocorrência do estado da natureza $\mathrm{N}_{1}$, êle obterá um lucro líquido de $\$ 500$. Os demais pay-offs são determinados de forma análoga. Vamos supor, agora, que o administrador faça uma estimativa da probabilidade de ocorrência de $\mathrm{N}_{1}$ e $\mathrm{N}_{2}$ e que êsses valôres sejam, respectivamente, 0,10 e 0,90 . No entanto, êle não tem muita confiança na veracidade destas estimativas e deseja saber o quanto elas podem variar para que sua decisão não seja alterada. Se o administrador calcular o valor esperado de cada alternativa, irá encontrar:

$$
\begin{aligned}
& A_{1}=(\$ 500 \times 0,10)+(\$ 400 \times 0,90)=\$ 410 \\
& A_{2}=(\$ 700 \times 0,10)+(\$ 200 \times 0,90)=\$ 250 \\
& A_{3}=(\$ 600 \times 0,10)+(\$ 100 \times 0,90)=\$ 150
\end{aligned}
$$

Desta forma, sua melhor ação será escolher a alternativa $A_{1}$, que the dá o maior valor esperado. No entanto, como êle não tem muita confiança nestas probabilidades estimadas, devemos determinar qual 
a faixa de variação a que estão sujeitas, de tal forma que êle não altere sua decisão, isto é, continue preferindo a alternativa $A_{1}$.

Se chamarmos $\mathrm{p}$ a probabilidade de ocorrência de $N_{1}$, e $(1-p)$ a probabilidade de ocorrência de $\mathbf{N}_{2}$, para que $A_{1}$ seja preferível a $A_{2}$ deveríamos ter:

$\$ 500 \times \mathrm{p}+\$ 400(1-\mathrm{p})>\$ 700 \times \mathrm{p}+\$ 200(1-\mathrm{p})$ donde $\mathrm{p}<1 / 2$

Isto significa que sempre que a probabilidade de $\mathrm{N}_{1}$ fôr menor que 1/2, a alternativa $A_{1}$ será preferível à alternativa $A_{2}$.

Da mesma forma precisamos verificar a preferência de $A_{1}$ em relação à $\mathbf{A}_{2}$, ou seja,

$\$ 500 \times p+\$ 400(1-p)>\$ 600 \times p+\$ 100(1-p)$ donde $\mathrm{p}<1 / 2$

Isto significa que sempre que a probabilidade de $\mathbf{N}_{1}$ fôr menor que $3 / 4$, a alternativa $\mathbf{A}_{1}$ será preferível à alternativa $\mathbf{A}_{2}$.

Com êsses dados em mãos, o administrador pode reavaliar as estimativas das probabilidades de $N_{1}$ e de $N_{2}$ que não lhe inspiravam grande confiança. Agora, êle pode dizer que a alternativa $\mathbf{A}_{1}$ será preferível a $\mathbf{A}_{2}$ e $\mathbf{A}_{3}$ caso a probabilidade de ocorrência de $\mathbf{N}_{1}$ seja inferior a $50 \%$ (e não mais igual a $10 \%$ como fôra estimado anteriormente).

\section{Conclusão}

O exemplo seguido, embora bastante simples, apresenta quase todos os estudos possíveis através da aplicação da Teoria da Decisão Bayesiana. Omitimos, propositadamente, a análise preposteriori, para que o exemplo não se tornasse demasiadamente complexo. No entanto, devido a sua real importância julgamos que êste aspecto da abordagem bayesiana deveria ser analisado à parte, possivelmente em um próximo artigo...

No decorrer do presente trabalho fizemos alguns comentários com relação à análise a priori, complementando-a com a análise de sensitividade, instrumento que permite ao administrador uma maior flexibilidade na estimativa das probabilidades a priori. Trabalhamos também com a análise a posteriori, através da aplicação do Teorema 
de BAYEs, que nos possibilitou a alteração das probabilidades a priori através da obtenção de informações adicionais. Finalmente, todo o contexto do exemplo apresentado estêve inserido numa série de decisões seqüenciais, fato êste típico da grande maioria das decisões mercadológicas. Estes elementos, em seu conjunto, constituem os componentes da Teoria de Decisão Bayesiana, como tivemos oportunidade de verificar no presente trabalho.

Como palavra final, gostaríamos de citar algumas áreas mercadológicas nas quais se desenvolveu com bastante êxito a abordagem bayesiana: problemas de determinação de preço, ${ }^{6}$ problemas referentes ao composto de comunicações, ${ }^{7}$ problemas ligados ao composto de apresentação, ${ }^{8}$ e problemas de pesquisa de mercado. ${ }^{9}$

Face à grande ênfase que vem sendo dada à abordagem quantitativa na mercadologia e, levando-se em consideração o poderoso instrumento que é a abordagem bayesiana, é de se esperar que seu uso venha auxiliar, cada vez mais, o administrador no difícil encargo de tomar decisões, o que é a própria essência da administração.

6 Green, P. E. Bayesian Decision Theory in Pricing Strategy, Journal of Marketing, january 1963 , p. 5 e segs.

7 Green, P. E. \& Frank, R. E. Quantitative Methods in Marketing, PrenticeHall, Inc., 1967, p. 10 e segs.

8 Alderson, W. \& Green, P. E. Op. cit., p. 108 e segs.

9 Myers, J. H. \& SAMLI, A. C. Management Control of Marketing Research, Journal of Marketing Research, agôsto de 1969. 